Portland State University

PDXScholar

1985

\title{
Scotopic and photopic thresholds : a dependent variable for the McCollough effect
}

Marilyn Murphy

Portland State University

Follow this and additional works at: https://pdxscholar.library.pdx.edu/open_access_etds

Part of the Cognition and Perception Commons

Let us know how access to this document benefits you.

\section{Recommended Citation}

Murphy, Marilyn, "Scotopic and photopic thresholds : a dependent variable for the McCollough effect" (1985). Dissertations and Theses. Paper 3529.

https://doi.org/10.15760/etd.5413

This Thesis is brought to you for free and open access. It has been accepted for inclusion in Dissertations and Theses by an authorized administrator of PDXScholar. Please contact us if we can make this document more accessible: pdxscholar@pdx.edu. 
AN ABSTRACT OF THE THESIS OF Marilyn Murphy for the Master of Science in Psychology presented November 22, 1985.

Title: Scotopic and Photopic Thresholds: A Dependent Variable for the McCollough Effect.

APPROVED 3Y MEMBERS OF THE THESIS COMMITTEE:

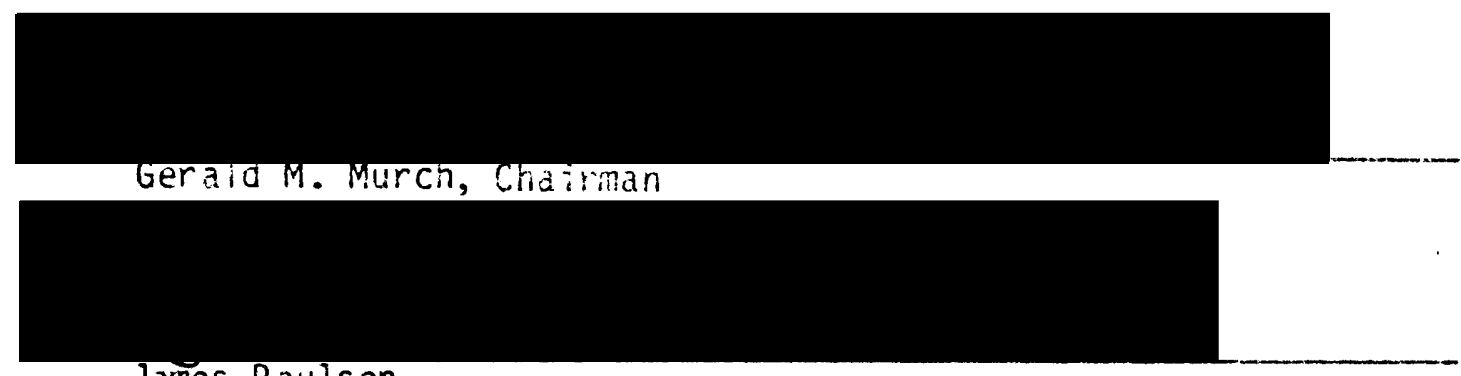

James Paulson

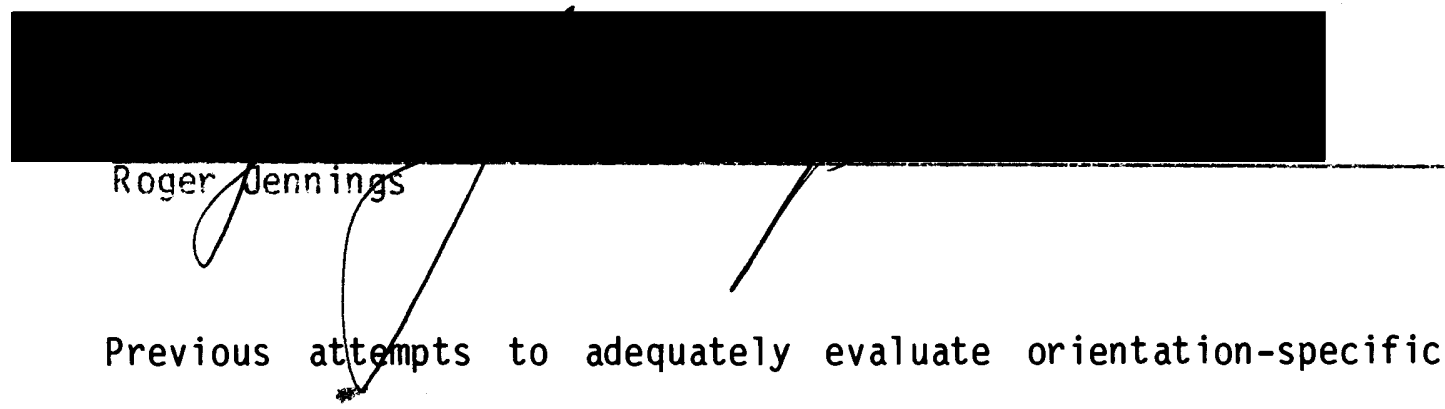

colored aftereffects (McCollough Effects) have thus far been unsuccessful in yielding quantitative measures of relative strengths of the effects. Similarly, little success has been shown in previous attempts to establish what level of illumination is necessary in order to evoke the aftereffects. The present study sought to determine (a) whether threshold measurements could serve as adequate, quantitative dependent variables of the McCollough Effects, (b) whether photopic illumination was necessary in 
order to evoke the aftereffect. It was predicted that the greater the number of inspection trials, the dimmer the $\$ 1 l$ lumination level could be in order for the aftereffects to first be detected. In addition, it was predicted that the appearance of the test pattern would change markediy near the rod-cone break. The strength of the McCollough Effects, as a function of the number of inspection trials, was examined on subsequent scotopic and photopic threshold measurements in normal color-visioned subjects. All subjects were measured alternately on 10 ascending and 10 descending trials at specific times after being conditioned in "short" (15 $\mathrm{min})$ and "long" (30 min) inspection trials, counterbalanced across subjects, with a 7-day interval between test trials. 0verall, statistically insignificant results were achieved for both predictions; possible explanation of the results and changes in experimental design were discussed. 
SCOTOPIC AND PHOTOPIC THRESHOLDS:

A DEPENDENT VARIABLE FOR THE MCCOLLOUGH EFFECT

$$
\text { by }
$$

MARILYN MURPHY

A thesis submitted in partial fulfillment of the requirements for the degree of

MASTER OF SCIENCE

PSYCHOLOGY

Portland State University

1985 
TO THE OFFICE OF GRADUATE STUDIES AND RESEARCH:

The members of the Comnittee approve the thesis of Mariiyn Murphy presented November 22, 1385.

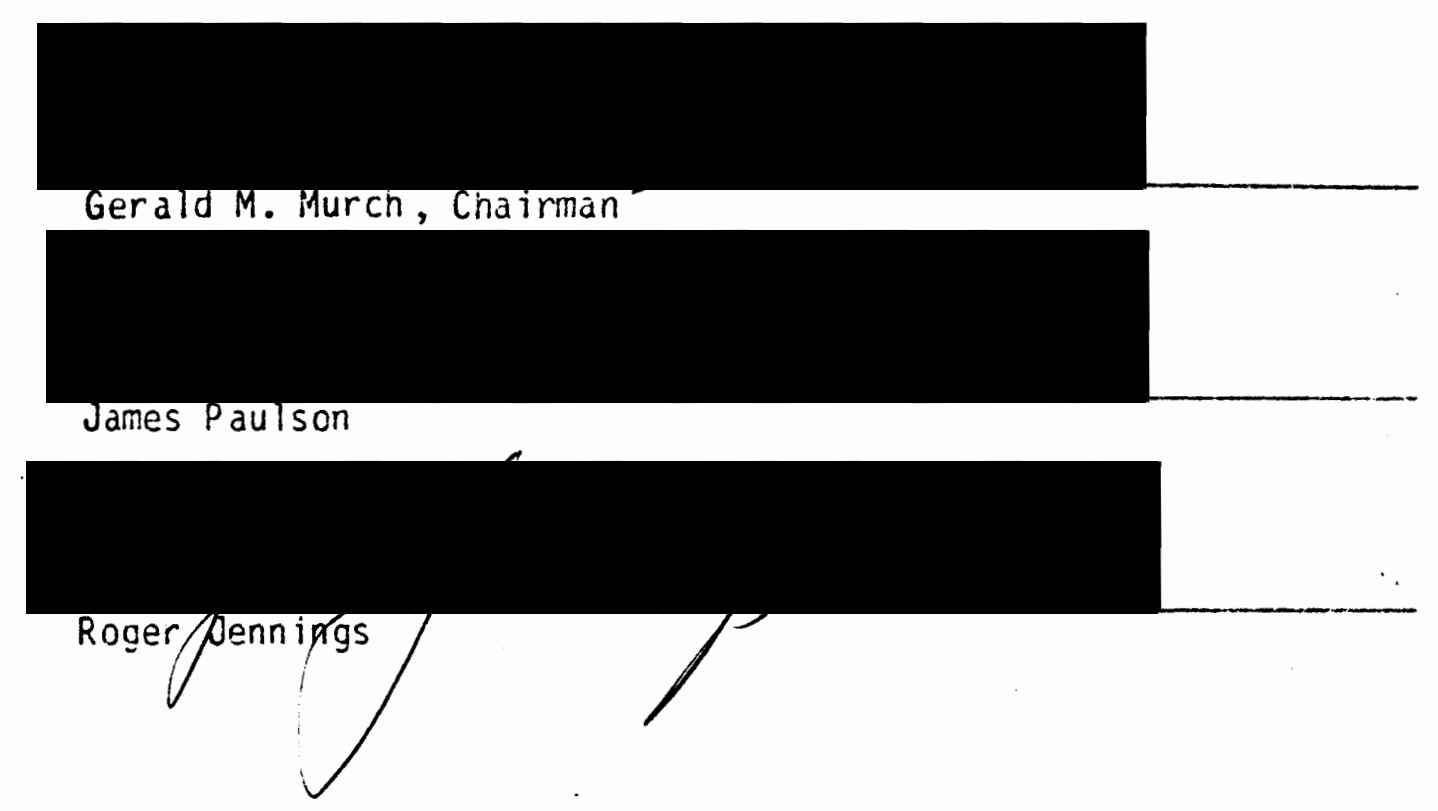

APPROVED:

Roger Zennings Head, Department of Psychology

Jim F. Heath - Vean of Graduate Studies and Research 
TABLE OF CONTENTS

PAGE

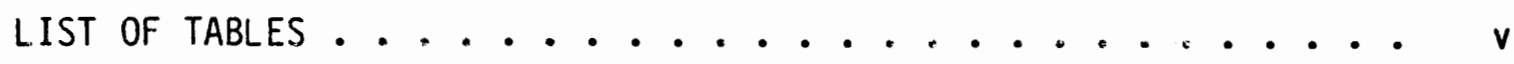

\section{CHAPTER}

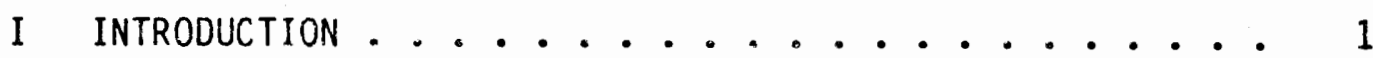

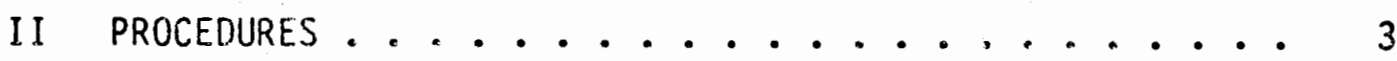

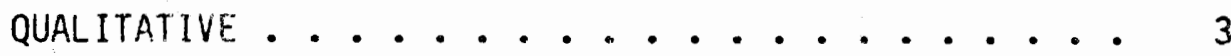

Unrestricted Colour Naming. ......... 3

Forced-Choice Colouring Naming Procedure. . . . 3

Colour-cancellation ........... 4

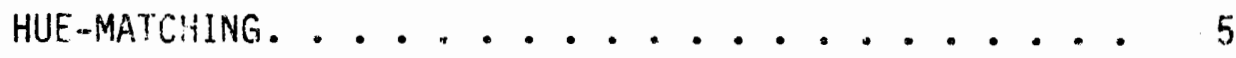

QUANTITATIVE. ................ 6

Magnitude Estimation........... 5

EVALUATION OF PAST RESEARCH EMPLOYING THRESHOLD

MEASUREMENTS. ................7

Spatial features. . ........... 7

Luminance Features. ............ 11

Temporal $\mathrm{P}$ arameters .......... 12

II: limitations. . . . . . . . . . . . . 16

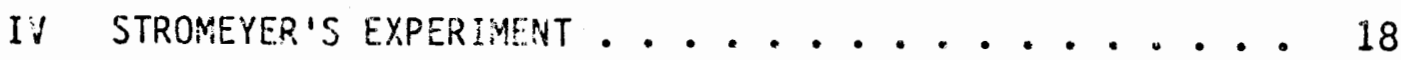

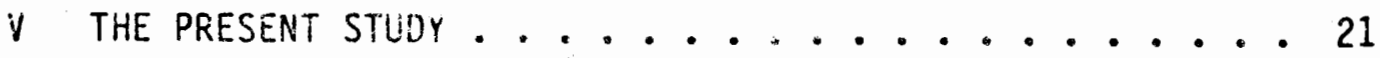

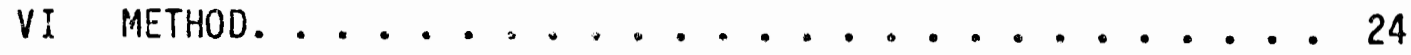

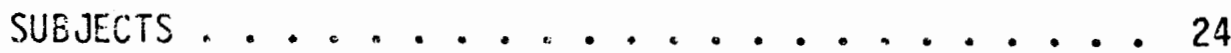

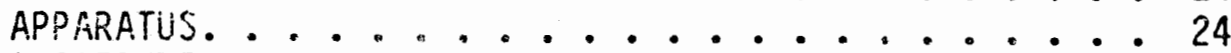

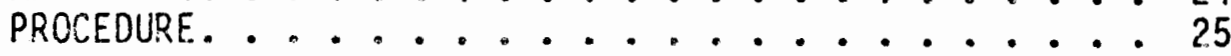

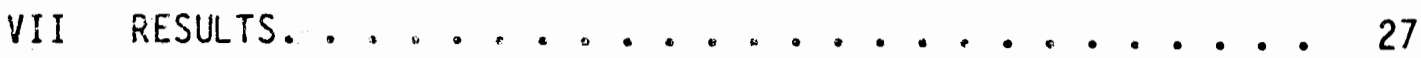

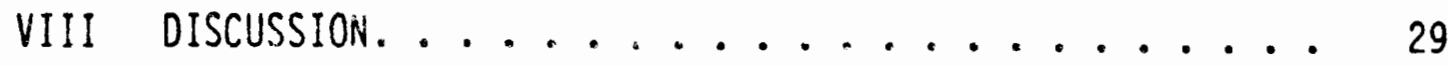


CHAPTER

PAGE

REFERENCES • . . . . . . . . . . . . . . . . . . . . 36 


\section{LIST OF TABLES}

TABLE

PAGE

I Raw data. . . . . . . . . . . . . . . . 34

I Means and standard deviations . . . . . . . . . 35 


\title{
CHAPTER I
}

\author{
INTRODUCTION
}

\section{SCOTOPIC AND PHOTOPIC THRESHOLDS}

\section{A Dependent Variable for the McCollough Effect}

The discovery of chromatic aftereffects which are contingent upon the orientation of lines was first described by McCollough (1965). In her original study, observers viewed 1 ine gratings, with one orientation paired with orange and the orthogonal orientation paired with the complementary color, blue. Upon inspection of an achromatic test pattern having both orientations of lines, subjects subsequently reported the test pattern as having color differences, with the color of the aftereffect contingent upon test orientation. Specifically, the orange verticallylined grating viewed during inspection yielded aftereffects tinged with blue, and the blue horizontally-lined inspection figure produced aftereffects tinted with orange.

Since that time, a multitude of variations on the original experiment demonstrating the effect have been reported (see for reviews Harris, 1980; Skowbo, 1984; Skowbo, Timney, Gentry and Morant, 1975), and although it has been established repeatedly that the color-contingent aftereffects are rather simple to evoke, finding a way to adequately quantify them has proven a much more challenging and difficult task (Skowbo et al.. 1975). That is, most measures simply identified the presence or absence of the aftereffect without providing a means of assessing the magnitude of the 
effect. Thus the impact of variations in independent variables have proven difficult to evaluate. The following summarizes the qualitative and quantitative procedures used to evaluate the McCollough Effect. 


\section{CHAPTER II}

PROCEDURES

\section{QUALITATIVE}

\section{Unrestricted Colour Naming}

Unrestricted color naming, the method McCollough (1965) employed in her original experiment, involved asking subjects after several inspection trials whether or not they observed any differences in hue, no matter how desaturated, between the two halves of the test grid. Next they were asked to write down any color differences they perceived. This procedure was carried out first for the left eye alone, then the right eye alone, for binocular viewing, and finally by alternately opening and closing each eye.

\section{Forced-Choice Colour Naming Procedure}

Harris and Gibson (1968) utilizing a forced-choice color naming procedure, required that every observer answer after each test trial, a set of five questions read to him from a printed checklist by the experimenter. Questions asked were: (a) "Do you see any color on the center diamond? (b) "If you had to pick a color for the center diamond, would you call it green, or pink? You must answer this question even if you don't see any color" (p. 1507). Next, the same two questions were 
asked about the diamond surrounding the center diamond. Lastly, each experimental participant was asked whether the color observed (if any) seemed stronger, the same, or weaker than the color observed on the immediately preceding test trial. The subject was then asked to rotate his head to the side 90 degrees and answer the same five-question check 1 ist (p. 1507).

Colour-cancellation

Colour-cancellation, a classification encompassing several varied procedures, involved manipulating some aspect of the dependent variable until the aftereffects disappeared. Teft and Clark (1968) measured the degree of rotation from adaptation orientation necessary for each subject to report the disappearance of the apparent color from the test grating. Riggs, White and Eimas (1974) designed an experiment whereby each observer was required to manually adjust a disk controlling variable amounts of desaturated light which in their combination with the test grating, eliminated the aftereffect. Over five trials for each pattern, a colorimetric purity index was derived; the amounts of light needed by each subject to just cancel the color on the test screen yielded an estimate of the presence and colorimetric direction of the aftereffect.

The three dependent measures described thus far: unrestricted color naming, forced-choice naming, and color cancellation procedures, having yielded qualitative assessments of the McCollough Effects establishing whether or not the colored aftereffects were indeed visible on the testing grid after the usual inspection sequences. 
HUE-MATCHING

Researchers who employed hue-matching as the dependent variable of choice began to span the distance between qualitative and quantitative assessments determining not only the presence or absence of the effects, but attempting to evaluate the strengths of the effects relative to each other. In Stromeyer's (1969) design, following several inspection trials, subjects were requested to match Munsell colored papers with the color of the aftereffects perceived on the test grating. The Munsell (1941) system of color notation consists of a set of colored paper chips arranged such that they are just noticeably different from each other in terms of hue, saturation, and brightness. Arrayed on the pages of a book, each page represents a different color, with saturation ordered along the horizontal dimension of each page and brightness along the vertical dimension. An experimental participant is then able to identify an unknown color experience by matching it to the Munsell chip it most closely approximates. Thus, both the direction of the color (qualitative) and the magnitude of the color (quantitative) can be evaluated, the former given by hue identification and the latter given by the degree of saturation.

Using a somewhat different process, Stromeyer (1972), by use of a rotating polarizer, required subjects to manually adjust the saturation of a projected field of color of similar hue to that of the test grating, to match the strength of the color observed on the test grating.

Murch and Hirsch (1972), after asking subjects to first $n$ ame the color they observed on the test gratings, rendered useful hue-matching 
by means of a projection colorimeter. This enabled them to measure the amount of each primary wavelength required by the subject to match the color of each of two test fields. These colorimetric values were then converted to CIE $x, y$ coordinates. The CIE (International Commission on Illumination) was established in 1924 to adopt a standard colorimetric system, dictated by the facts of color mixture using $X$ (red), $Y$ (green), $Z$ (blue), as the three primary wavelengths (Wasserman, 1978).

A tristimulus projection colorimeter was used in Skowbo, Gentry, Timney and Morant's (1974) experimental design; after subjects were presented with the usual McCollough Effect adaptation sequence, they were then exposed to five post-adaptation stimulation (PAS) conditions. In order to measure the impact of the PAS states upon the rate of decay of the aftereffects, a homogeneous chromatic field was presented to the right eye. Subjects were asked to adjust the wavelength contributions in order to match the color on the test gratings viewed with the left eye. The obtained values were then converted into CIE chromaticity coordinates. Several other investigators have used a similar approach (Hirsch \& Murch, 1972; Mack ay \& Mack ay, 1977; Murch, 1979, Timney, Gentry, Skowbo \& Morant, 1974) in attempting to quantify the McCollough Effects.

QUANTITATIVE:

Magnitude Estimation

Stromeyer's (1969) paradigm for determining the relative strengths of the aftereffects involved asking each subject to assess the saturation of the first aftereffect viewed during the test phase by assigning to it 
an arbitrary number. Each successive aftereffect was then rated in proportion to the first number, thereby yielding a magnitude estimation for each aftereffect. This procedure marked the transition from qualitative to quantitative dependent measures of the McCollough Effects.

EVALUATION OF PAST RESEARCH EMPLOYING THRESHOLD MEASUREMENTS:

\section{Threshold Measurements}

In the continuing search for quantitative dependent variables of the McCollough Effects, it was found that by systematically manipulating various features of the independent variables (spatial, luminance and temporal parameters of the inducing and testing grids, and using the aforementioned techniques to gauge them) far more informative threshold measurements of the strengths of the aftereffects relative to each other could be obtained. Thus, researchers were now able to quantify, by altering such variables, the minimum stimulus energy necessary for an observer to indicate an awareness of stimulus change (Wolman, 1973, p. $386)$.

\section{Spatial Features}

Variations in spatial features of the inducing and test grids were extensively pursued. In light of Hubel and Wiesel's (1968) findings that electrical stimulation applied to certain cells located within the striate cortex of the cat revealed orientation specific units with a high degree of angular specificity, and results from experiments with monkeys stimulated in the same cortical region yielding cells even more selective 
to orientation, Fidell (1968) postulated that perhaps within the human visual system there could also be edge-detecting units selectively sensitive to different orientations of lines. The McCollough Effect would therefore be evoked only if the orientation of lines on each pair of adapting grids (paired with complimentary colors) were divergent enough from each other to stimulate different populations of cortical units. Fidell attempted then to arrive at a quantitative index of (a) the minimum angle of divergence between adapting stimuli necessary to produce the effect and, (b) the intensity of the effect at different angles of divergence. This was accomplished by recording each subject's verbal color-detection response ("hue" or "no hue"), then their verbal forcedchoice color naming response ("red", "green", "blue" or " "yellow") after each presentation of the test stimulus. To measure the strengths of the effects, experimental observers were required to manually adjust an unmarked dial which varied the saturation, or colorimetric purity, of the test pattern in order to equalize each to a neutral color. Pre- and postadaption readings were taken on the unpatterned control stimulus to measure the amount of generalized color adaption (usually negligible) on each half of each patterned test stimulus.

Not only were orientation thresholds obtained as possible determinants of the intensity of the effects, but "density" thresholds as well. Teft and Clark (1968), using the color-cancellation procedure discussed earlier, examined the relationship of the density of lines between adapting and test patterns. By varying the density of lines, or number of edges between adapting and test patterns, they were able to measure the degree of rotation from adapting orientation necessary for 
the subject to report the disappearance of the apparent color from the test field.

Supported by physiological findings from animal research (Hubel and Wiesel, 1968) Lovegrove and Over (1972) presented observers with the usual McCollough Effect adaptation sequences varying however, the spatial frequencies between the vertical and horizontal inspection grids. Subjects were then instructed to report verbally whether or not either half of the test display appeared colored, and if it did, would the color most approximate green or red; the forced-choice color naming procedure employed by Harris and Gibson (1968).

Leppmann (1973), utilizing inducing grids of differing spatial frequencies, was able to demonstrate the corresponding color-conditioned aftereffects using a variable-frequency test grid. By obtaining verbal, hence qualitative, estimates of (a) the appearance of colors on the test field and (b) the saturation of the colors on the test grid relative to each other, he was able to show the strongest effects on the portion of the variable-frequency test field that most closely resembled the spatial frequency of the inducing grid.

Riggs (1973), using pairs of oppositely curved inspection grids projected on red and green colored backgrounds respectively, was able to induce the subjective colors using achromatic test fields composed of alternating patterns of convex/concave lines. Observers were asked to verbally indicate whether or not there seemed to be any hue differences on the test panels.

Following this, conditioning continued until subjects were able to correctly name the appropriate color for each particular curvature. 
Regardless of the degree of curvature observed on the grids during conditioning trials, the most vivid aftereffects were reported on those test fields with the most strongly curved panels, and viewing inspection patterns with stronger curvatures yielded McCollough Effects more rapidly than did viewing grids composed of more weakly curved lines.

Demonstrating results of a similar nature, White and Riggs (1974) produced the effects using inspection panels composed of oppositelypointed chevron ( $V$-shaped) patterns. Presented with achromatic test grids, experimental participants were asked to verbally judge the appearance of any color present on any portion of the grid. If any color was reported, they were then asked to specify what portion of the test pattern appeared colored and to name the color. The McCollough Effects were most easily induced when the lines of the chevron patterns used during inspection formed 90 degree angles.

Following the usual adaptation sequences, Sharpe and Tees (1978) measured the strength of the subjective aftereffects using magnitude estimations by asking subjects to verbally respond to the following questions when presented with the testing stimuli:

Do you observe any color differences between the two halves? If so, assign simple color names to the different halves; then assign a value of five on a scale from zero to five to the strongest aftereffect elicited, and scale all subsequently presented test patterns (p. 452).

Using inducing grids composed of segmented or blurred lines, observers reported the strongest colored aftereffects on test panels with 
complete or focused lines: the magnitude of the effects decreased as the similarity of the inspection and test grids decreased.

\section{Luminance Features}

While not as widely investigated as other possible parameters of the effects, systematic manipulations of luminance features of the adapting and testing fields were concurrently pursued. Stromeyer and Mansfield (1970), the first investigators to provide information about the luminance parameters of the inducing grids used to evoke the subjective colors, employed an unrestricted color naming procedure. They determined that rather than the effects being dependent on some absolute level of adaptation illumination, marked decreases in luminance levels of the inspections grids could occur without there being a corresponding change in the aftereffects.

Following brief ( $5 \mathrm{sec}$ ) and prolonged (15 sec) adaptation to horizontally or vertically lined grids paired with varying background colors, Timney, Gentry, Skowbo and Morant (1975) measured luminance thresholds for test fields of identical or differing orientation and wavelength. After inspection trials, observers were required to manually adjust a neutral density wedge until such time that the subjective colors were just apparent on the test target. Brief viewing durations yielded slight elevations in orientation-specific luminance thresholds while prolonged adaptation produced larger orientation specific thresholds.

Using a color-cancellation technique, white (1976) revealed that over inspection trials of equal duration, stronger effects were induced using high luminance adaptation gratings rather than lower luminance 
gratings and stronger effects were viewed on lower luminance test fields than higher luminance fields.

Investigating pattern contrast, Schmidt and Finke (1979) reported that stronger subjective colors were observed on high-contrast test patterns, the strength attributable to the high degree of luminance contrast present within each test target rather than to similarities in luminance levels between inspection and testing grids. The effects were assessed using a forced-choice procedure; participants were requested to "inspect the pattern for at least several seconds and then, when ready, write down on the prepared form which half of each test slide appeared "redder" or "greener" (p. 409). Directly following each experimental session, subjects were encouraged to write a short description of their observations and then discuss them briefly with the investigator.

Using a similar procedure, Mikaelian (1980) determined that highcontrast adaptation figures yielded subjective reports of stronger McCollough Effects than did low-contrast inspection fields.

\section{Temporal Parameters}

Variations in temporal parameters were methodically investigated. Looking at rates of acquisition of the McCollough Effects, Stromeyer (1969) first reported that the rate of alternation between inspection and test figures seemed to have little effect in evoking the subjective colors on stationary test gratings. Skowbo and Rich (1982) using a hue-matching assessment procedure, strove to relate relative strengths of the aftereffects to the length of exposure to adapting fields. Their results, plotted on an acquisition curve, revealed a steep rise in strength 
initially, followed by more moderate increments in strength. In a related view, Skowbo and White (1983) manipulated trial duration and the number of alternations of adapting grids within trials. Statistically significant results were achieved for trial length only; the number of color/contour pairings had no significant effect.

Longevity and rates of decay of the colors were similarly pursued. That they could last as long as six weeks after a 20 min inspection sequence was demonstrated by Stromeyer and Mansfield (1970). Riggs et a). (1974) have noted increases in the magnitude of the effects with adaptation times of up to $150 \mathrm{~min}$ and Mackay and Mackay (1974) showed strengthening of the colors after periods of sleep. Pursuant to Mackay and Mackay (1974) results, Lund and Mackay (1983) found that subjects could lose up to $50 \%$ of initial strength of the aftereffects, if deprived of three to four $\mathrm{hr}$ of sleep the $\mathrm{night}$ preceding experimental sessions. Finally, other researchers have manipulated the temporal relationship between contour/color pairings during inspection sequences, attempting to shed light on a mechanism or underlying model to explain the effects. As the physiological basis of the McCollough Effect is at present unknown, two theoretical models have emerged, each attributing the colors to different processes occurring within the visual system. One class of models assumes a static, "prewired" visual system, whereby color-coded edge detectors undergo chromatic adaptation (a change in sensitivity) after being exposed to the usual McCollough Effect inspection sequences. The second class of models credits the aftereffects to a conditionling, or learning process during which the background color of the inspection panel serves as the UCS and the lined grading as the CS. 
After repeated pairing, the lined grid alone functions to evoke the colored aftereffects, the CR.

Murch (1976) pursuing this line of reasoning, attempted to explore the temporal relationship between the UCS and the CS, employing both a delay and trace conditioning paradigm. Using magnitude estimations ranging from negative numbers if the test stimulus appeared "pinkish", to positive numbers if the test target appeared "greenish" to assess the strengths of the effects, he demonstrated that the temporal parameters of the effects conformed to known attributes of the classical conditioning model. A forced choice color naming procedure was utilized by Schmidt, Pinette and Finke (1978); they required observers to verbally report which half of the test target, after viewing the usual adaptation figures, appeared more red. Results from three experiments, using different temporal orders during inspection, were interpreted as yielding further evidence of a classically-conditioned learning process.

Skowbo and Forster (1983) based their research design on experimental findings extracted from Rescorla and Holland's (1982) recent review:

...close temporal contiguity between the CS and UCS would fail to produce conditioning if, during acquisition trials, the UCS were also presented frequently without the CS: Such a maneuver would lower the correlation between CS and UCS occurrences (cited in Skowbo, 1984).

In a similar fashion, Skowbo and Forster (1983) designated the colored panel as the UCS and the lined grating as the CS, interspersed homogeneous colored stimuli in random fashion, in the usual inspection sequences. Their results were interpreted as going against Rescorla and Holland's (1982) predictions, in that reported strengths of the 
aftereffects were not weaker than those in the control condition, where homogeneous achromatic panels were used in place of the chromatic homogeneous panels.

From results cited in her most recent review of the literature, Skowbo (1984) concluded that while many similarities have been demonstrated between the basic mechanisms of conditioning and the McCollough Effects, until it can be empirically verified as to what conditions are necessary as well as sufficient for evoking the effects, the basic mechanism by which the subjective colors are evoked remains in question.

In a reply (Holding, in press) to Skowbo (1984), it was stated that she based her assertion, that "McCollough Effects are not classically conditioned responses" ( $p .215$ ) on classical conditioning literature that was somewhat out of date. Since the time of her publication, Brand, Holding, and Jones (in press) have investigated the susceptibility of the McCollough Effects to the phenomenon of blocking, defined by Kamin (1969) as ...occurring when prior conditioning to one stimulus prevents or impairs the subsequent, simultaneous conditioning of a second stimulus (cited in Brand et al., in press). Using color matching as the assessment procedure, they were able to successfully demonstrate blocking of the conditioned aftereffects and regarded these results as yet another piece of evidence concurring with a learning model underlying the effects. 


\section{CHAPTER III}

\section{LIMITATIONS}

Albeit the majority of these potential yardsticks of the McCollough Effect have been successful in establishing an intimate relationship between color and contour mechan isms, most measures have serious drawbacks and limitations that make quantifiable interpretation and comparison of the data next to impossible.

Those researchers who emp loyed unrestricted color naming found that naive subjects were sometimes unable to report whether or not the colored aftereffects were visible on the test grating or were unable to attach color names to them, possibly due to the desaturated appearance of the hues (McCollough 1965). Running into difficulties of the opposite nature, Harris and Gibson (1968) noted that subjects who were unable to report the appearance of colors on the test field were nonetheless able to give suitable responses when forced to choose!

Objections have also been raised regarding the efficacy of certain color-cancellation procedures. Although Teft and Clark (1968) proposed to measure changes in test pattern orientation, Skowbo et al. (1975) commented that what was actually being measured was bandwidth; the range of spatial frequencies over which the effect remained visible.

It should also be noted that hue-matching used in cancellation experiments assumes a linear relationship between hue strength and aftereffect strength. That the visual perception of hue is a non-linear 
function is well documented (Murch, 1976), thus rendering this technique questionable.

Gauging the relative strengths or magnitude estimates of the McCollough Effects have yielded finer measures of the effects than the simple reporting of whether or not the appropriate hues were perceived. Placing constraints on this method however was the narrow range in magnitude (or degree of difference), over which the effects seem to occur (Stromeyer, 1969). 


\section{CHAPTER IV}

\section{STROMEYER'S EXPERIMENT}

Taking a new slant on the problem, Stromeyer (1974) found that formspecific colored aftereffects could be evoked from test patterns observed in extremely dim light that stimulated the rods but not the cones. His results suggested that rod and cone signals converge at some level of the visual system. Previous to this time, evidence indicated that rods alone functioned in dim light (scotopic illumination) so that objects appeared colorless with cones contributing input only at higher levels of illumination (photopic) to give objects color and form.

In regards to Stromeyer's (1974) controversial findings, an obvious question must be raised-- is there an absolute crossover from scotopic to photopic sensitivity and through conditioning could the strength of the McCollough Effect lower the threshold at which the effect could be perceived or is the McCollough Effect merely a function of some static, prewired constant, an observer's dark adaptation curve.

Another point of contention which must be mentioned but will not be dealt with extensively in this paper centers around the basic mechan ism by which the effect is evoked. The issue of identifying that mechanism has led to the development of two theoretical camps. One group, the "adaptation" theorists, ascribes the effect to some $k$ ind of physiological chromatic adaptation to specific components (i.e., lined grids of specific 
orientation) and to wavelength, while the opposing group, the "classical conditioning theorists", presumes a learning or associative mechanism by which the specific components acquire the capability of evoking the aftereffect.

The adaptation theories/ contend that the background color of the test pattern is the feature component used in producing the etfect. Contrarily, for the learning model, the lined grid serves as the initiating stimulus which evokes the aftereffect. The lined grid may be viewed as the CS which, when paired with the background color (UCS), evokes the conditioned response of adaptation, or a change in sensitivity.

Consequently, the adaptation model would predict that the light energy has to be sufficiently high for the background color to be picked up by both rods and cones, hence the Mccollough Effect could only be evoked in photopic illumination. On the other hand, the classical conditioning model would predict it should make no difference whether the illumination is scotopic or photopic in nature, as it is not the background color of the test pattern that is crucial, but the lined grid. (In fact, the McCollough Effect should be able to be evoked in total darkness if there was some vehicle for testing it!).

The fact that Stromeyer (1974) did indeed evoke color-contingent aftereffects under scotopic illumination prompted the following pilot study. The study replicated Stromeyer's (1974) basic design with the exception of omitting the dark adaptation measurements and included a pretest and three different inspection times. A bal anced incomplete block design was used, subjects were run, each on two of the three conditions and ascending and descending threshold measurements were recorded. It 
was predicted that the greater the number of inspection trials, the dimmer the illumination could be in order for the observer to first perceive the color on the test pattern as illumination of the test pattern shifted from completely dark to bright (ascending threshold measurements). Likewise, the greater the number of inspection trials, the longer would the observer be able to still perceive the color on the test pattern, even though illumination of the test pattern was getting progressively dimmer (descending threshold measurements). The failure of the study to indicate a clearcut relationship between strength of the McCollough Effect and threshold measurements was attributed to extremely high measurement error. 


\section{CHAPTER $V$}

\section{THE PRESENT STUDY}

The present study, while attempting to closely parallel Stromeyer's (1974) design (in which a between-subjects format was used and ascending threshold measurements were obtained after a 20 min inspection trial), contained the following changes: A within-subjects design was employed, and ascending and descending threshold measurements were taken on each subject on both "short" (15 $\mathrm{min})$ and "long" (30 min) inspection times.

With these changes, the study sought to determine (a) whether threshold measurements could serve as quantitative dependent measures with which to evaluate the strength of the aftereffects and (b) whether the McCollough Effect required a photopic level of illumination in order to be evoked. Conversely, could the aftereffects be elicited in scotopic illumination? In accordance with (a), it was predicted that the greater the number of inspection trials, the dimmer the illumination could be in order for the subject to first perceive the desaturated hues on the test target. In accordance with (b), it was predicted that the appearance of the test pattern would change markedly near the rod-cone crossover point: just above the break the bars of the grating would appear sharp, and just below the break they would appear diffuse. The vertical and horizontal grating of the testing grid would appear faintly pinkish and greenish respectively at the lowest light level, minus $1.89 \log \mathrm{mL}$ below the rod- 
cone break and become progressively more saturated as the light was increased.

In relation to the predictions of the adaptation model, a photopic level of illumination would be necessary in order to observe the desaturated hues, since it was the chromatic background that was critical in evoking the effect. Contrarily, the classical conditioning model would anticipate that the greater the number of inspection sequences, the dimmer the illumination could be in order for the observer to first detect the colors on the test field, as it was the lined grid that served as the inducing stimulus, hence the McCollough Effects could be elicited in either photopic or scotopic illumination. Should the following study demonstrate that these colored aftereffects could be observed in scotopic, and well as photopic illumination, further evidence would be rendered concurring with the predictions of the classical conditioning model and refuting the predictions of the adaptation model .

Interestingly enough, not only does the pursuit of an adequate quantitative dependent variable generate interest within the academic community, but has particular relevance within the industrial setting as well-with regards to video display terminals with green phosphors. According to Long (1984), human factors psychologists have wrestled with the problem of what to do when operators of video display terminals with green phosphors report observing persistent pink-tinged aftereffects after viewing the screen.

Should the following study confirm the predictions stated previously, invaluable practical as well as theoretical contributions would be forthcoming, not orily expanding our understanding of mechanisms 
operating within the human visual system, but providing researchers in industry with the tools to thereby minimize or eliminate such effects. 


\section{CHAPTER VI}

METHOD

SUBJECTS

Twelve naive, color-normal subjects, as ascertained by HRR Plates, from the perception class at Portland State University participated in the experiment. Each was awarded three extra-credit points towards the final grade upon completion of the experiment.

APPARATUS

During the inspection phase, light from one projector was filtered

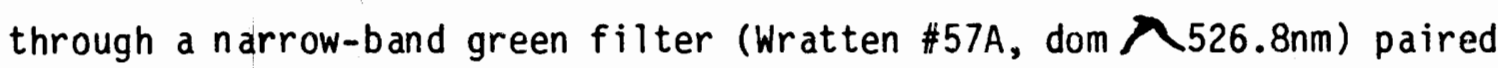
with a black vertically-lined square-wave grating while light presented alternately from the other projector was filtered through a complementary red filter (Wratten \#30, dom 入 $505.5 \mathrm{~nm}$ ) paired with a black horizontallylined square-wave grating; both gratings measuring 1.5 cycles per degree and subtending an area of 11 degrees square. A \#3 neutral density filter was placed in front of the lens on each inspection projector.

During dark adaptation measurement, light from the projector was passed through a left and right diagonally-lined black grating, 1.5 cycles per degree. 
During the test phase, the projector was mounted in such a way to allow the test beam to project through the test pattern and the variable neutral density wedge. The left portion of the test pattern consisted of a horizontally-lined grating contiguous with the right portion, a vertically-lined grating, both 1.5 cycle per degree. (A low spatial frequency was used because below the red-cone break, gratings above 5 cycles per degree cannot be resolved). All apparatus was housed and all testing conducted in a small, windowless room painted flat-black.

PROCEDURE

During the inspection phase, subjects, individually run, viewed the high-contrast gratings projected upon a $56 \times 56$ white cardboard screen. Counterbalanced across subjects, one half of the observers, randomly assigned, were exposed to the "short" condition first: 2-sec on and 2sec off alternating presentation of the green vertical grating and the red horizontal grating for 15 min totaling 113 trials with mean luminance of the green and red inspection stimuli being 4.519 and $4.950 \mathrm{~mL}$ respectively. Then, after dark adapting for $30 \mathrm{~min}$, during which time a dark adaptation curve was obtained, an alternating series of 10 ascending and 10 descending threshold measurements of the test pattern was recorded for each subject, the luminance of the white areas of the test pattern ranging between $-1.89 \log \mathrm{mL}$ and approximately $-6.00 \mathrm{log} \mathrm{mL}$.

With a 7-day interval separating conditions, the group receiving the "short" inspection time first was then exposed to a "long" condition, $30 \mathrm{~min}$ of inspection constituting 226 test trials. The remaining half 
of the subjects were run in the opposite order. Subsequent threshold measurements were recorded for each condition.

During the dark adaptation phase of each condition, dark adaptation curves were obtained in that after strong light adaptation to a $\mathrm{PH} / 5$ clear flash bulb $20,000 \mathrm{~lm} \mathrm{sec}$, observers fixated on the left and right diagonally-lined grid projected on the screen and would adjust, by manual control, a neutral wedge controlling illumination level, so that the grating would always remain just below threshold. These materials had the same reflectance as the test pattern used during inspection. 


\section{CHAPTER VII}

\section{RESULTS}

Individual scores representing the mean neutral density wedge setting (level of illumination) for 10 ascending and 10 descending threshold measurements for each subject are presented in Table 1.

Insert Table 1 about here

Additionally, means and standard deviations for grid threshold measurements 7 min into dark adaptation and subjective color threshold measurements in $15 \mathrm{~min}$ and 30 min inspection trials are shown in Table 2.

Insert Table 2 about here

A t-test for related measures was performed to test whether threshold measurements could serve as quantitative dependent variables with which to evaluate the strengths of the aftereffects. Results indicated ( $t$ ( 8 ) $=1.72, \mathrm{p}<.10$, one-tailed) that with longer inspection times ( $30 \mathrm{~min}$ ) 
illumination levels were marginally lower than during shorter inspection times $(15 \mathrm{~min})$.

Finally, it can be noted that higher levels of illumination were required to reach subjective color threshold in both $15 \mathrm{~min}$ and $30 \mathrm{~min}$ inspection trials than were required to reach grid threshold $7 \mathrm{~min}$ into dark adaptation. Hence no scotopic effect was observed. 
CHAPTER VIII

\section{DISCUSSION}

Collectively, the results do not provide significant evidence to render threshold measurements, as determined by neutral wedge settings, adequate quantitative dependent variables with which to evaluate the strength of the McCollough Effects, nor does this research indicate that the McCollough Effects can be elicited in scotopic illumination.

In regards to the former prediction, because only marginally significant results were achieved during $15 \mathrm{~min}$ and $30 \mathrm{~min}$ inspection trials (subjects were able to detect the hues in only slightly less illumination-determined by wedge setting, in the 30 min adaptation sequence than in the 15 min adaptation sequence), it can be concluded that threshold measurements, determined by wedge settings, do not serve as adequate dependent variables. This is in part due to the extremely high error variance observed between subjects. Because of this, statistically significant differences in strength between the two inspection conditions may be masked, or a more thorough examination of neutral density wedge setting to determine threshold measurements may yield no significant relationship at all.

Next, results demonstrated that the present study was unsuccessfill in evoking the McCollough Effects in scotopic illumination. Great variability within-subject's dark adaptation curves were observed over 
time. To use them as a standard against which to evaluate other dependent measures would be erroneous. In addition, it was observed that when subjects were allowed free range to select wedge settings to where they could just detect the desaturated hues on ascending trials, they went immediately into the photopic range! The observers may well have been able to perceive the aftereffects in scotopic illumination but the hues are more easily observed in higher levels of illumination. This lends further evidence to the insensitivity of this dependent measure.

At this point, obvious questions must be raised surrounding Stromeyer's (1974) experimental findings. The first point of departure centers on Stromeyer's (1974) dark adaptation threshold measurements curves. Although this study employed a similar procedure for obtaining dark adaptation measurements, it was unsuccessful in replicating Stromeyer's (1974) results. One possible explanation may lie in the observation that Stromeyer (1974) assigned fixed points at which to take threshold measurements; to do so may be arbitrary and erroneous. Additionally, on ly one dark adaptation curve was obtained for each subject, and no index was provided during dark adaptatior measurements to indicate where an observer was with regard to time.

Next, in interpreting Stromeyer's (1974) results two issues must be examined. First, Stromeyer (1974) used three subjects upon which to support his predictions, generating group data on so small a sample size can lead to distortions of the data, obscuring what results actually were obtained. Next, of his pool of three subjects, two were experienced observers of the McCollough Effect. This could lead to possible confounding results, although with regards to this contention, the 
literature is equivocal: Skowbo and Rich (1982) found no evidence to support changes in aftereffect strength or rate of acquisition with practiced observers. Murch (1974) and Shute (1979) however, reported that only extremely brief exposure times were necessary for trained observers to report detection of the hues.

Although serious concerns have been raised in reference to the strength of Stromeyer's (1974) controversial findings, his results could never-the-less be explained in terms of the classical conditioning model. This model would predict that because the lined grating of the inspection figure viewed during adaptation serves as the feature component in eliciting the desaturated hues, the level of illumination in which the hues are evoked should make no difference. Specifically, the effects could be elicited in scotopic illumination provided that there was a vehicle through which it could be evaluated. That Stromeyer (1974) was able to demonstrate this neatly conforms to these predictions.

Contrarily, the predictions of the adaptation model would not be suitable in explaining Stromeyer's (1974) data, due to its contention that the illumination level would have to be sufficiently high in order for observers to detect the hues, as it is the background color of the adapting grid that serves as the feature component in producing the effect. Stromeyer's (1974) demonstration of McCollough Effects evoked in the scotopic range refutes this claim.

The present study can lend confirmation to neither group of models in that overall, statistically insignificant results were achieved. Thus neither Stromeyer's (1974) study, nor the preserit study are able to provide convincing evidence for either of the models, in either direction. That 
is, both are unable to concur with or refute either model, due to the reasons just discussed.

While the present study has demonstrated that threshold measures, as determined by neutral density wedge settings are inadequate in serving as quantifiable dependent variables with which to evaluate the strengths of the McCollough Effects, perhaps with improvements, i.e., other ways of evaluating threshold measures, future studies could render them more sensitive and hence, adequate.

Additionally, improvements are necessary in obtaining dark adaptation curves. In future research they need to display consistency over time for them to be considered useful in serving as a standard against which other dependent variables can be assessed. To remedy this, an item not mentioned by Stromeyer (1974) and very much worth examining, would be to have subjects verbally report the point at which the lined grid appeared clearly sharp and the point at which the grating appeared fuzzy and indistinct. This would allow investigators to ident ify where observers were in their dark adaptation curve(where their rod-cone break occurred). obtaining threshold measurements periodically during adaptation sequences might provide a more sensitive index of the rod-cone threshold, as well as providing a more sensitive index of the strength of the effect. Evidence has been put forth by Holding, (in press) to suggest that the first test trial is simultaneously the first extinction trial. Employing such a technique, this phenomenon could easily be detected.

Another item worth investigating centers around the spatial frequency of the testing grid. Perhaps the width of the bars of the 
gratings used in this study were too fine, making the rod-cone break indistinguishable. A low frequency grating might yield a more definitive rod-cone threshold.

With these changes in experimental design and procedure, threshold measurements may yet be regarded as adequate, quantitative dependent variables with which to evaluate the strength of the McCollough Effects. On the other hand, maybe the reason investigators have thus far had such difficulty in finding a quantitative measure of the effects is that the McCollough Effects are not quantitative effects at all, but qualitative ones. Perhaps there is a distinct cross-over point, below which there are no effects and above which, there are. Only future research can determine this. 


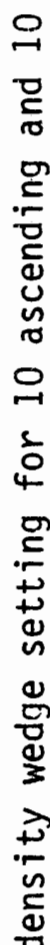

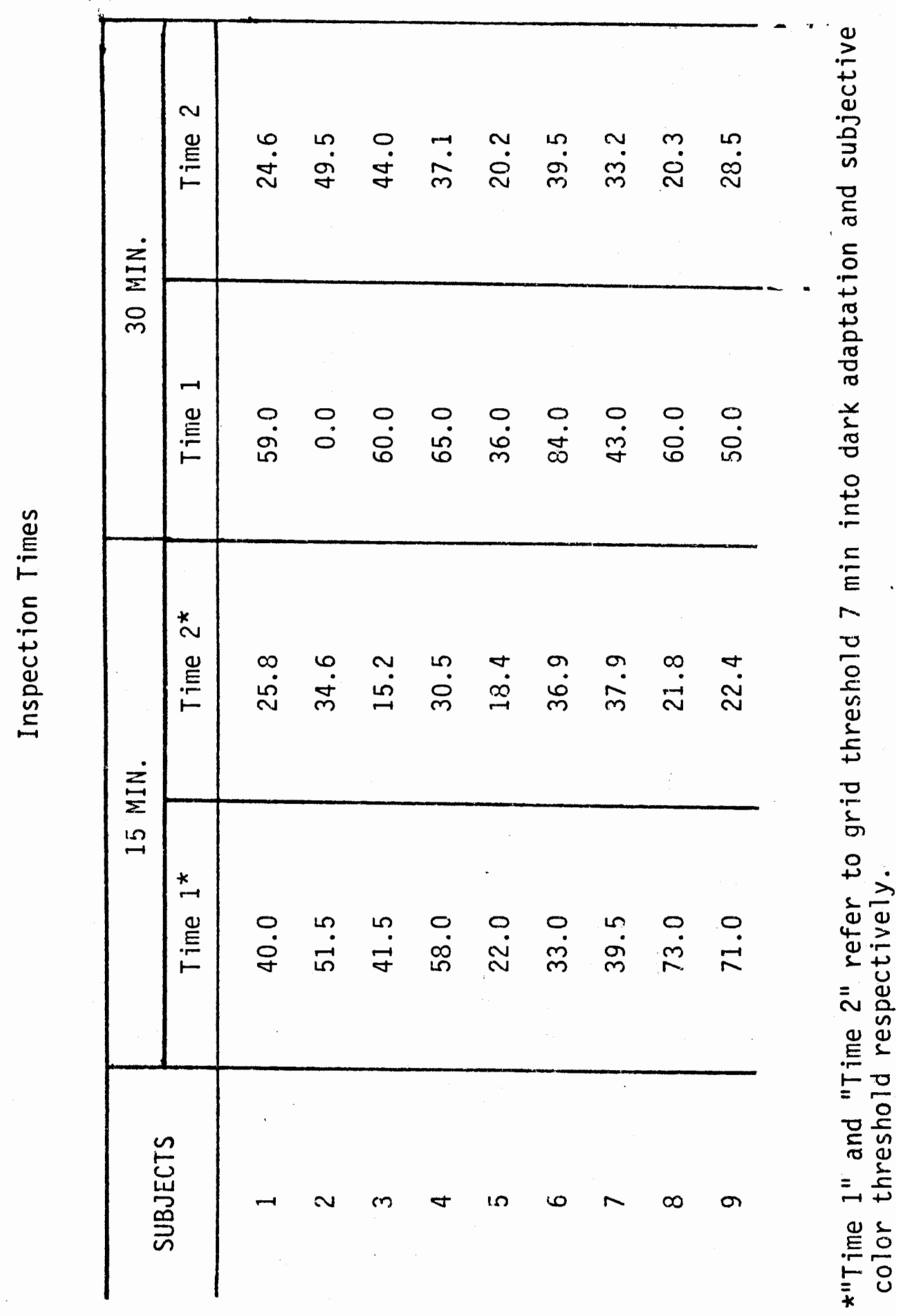



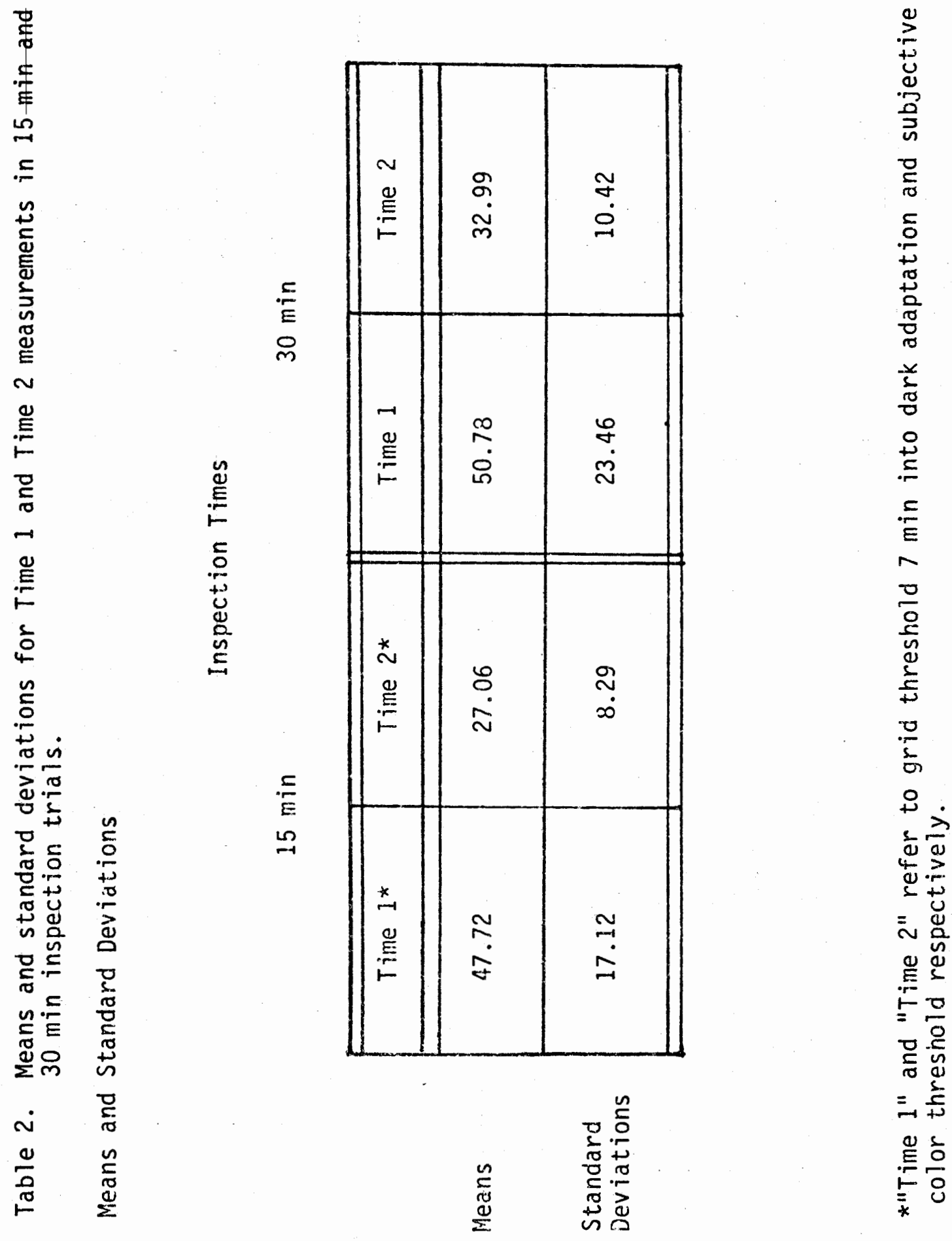
Brand, J.S., Holding, D.H., \& Jones, P.D. (in press). Conditioning and blocking of the McCollough effect. Perception and Psychophysics.

Fidell, L.K.S. (1968). Pattern-specific complementary hue aftereffects: more on the McCollough effect. Unpublished doctoral dissertation, University of Michigan.

Harris, C.S. (1980). Insight or out of sight? Two examples of perceptual plasticity in the humar adult. In C.S. Harris (Ed.) Visual coding and adaptability (pp 95-149), Hillsdale, M.J.: Erlbaum.

Harris, C.S., \& Gibson, A.R. (1968). Is orientation-specific color adaptation in human vision due to edge detertors, afterimages or "dipoles"?. Science, 162, 1506-1507.

Hirsch, J. \& Murch, G.M. (1972). Variation in hue of a contour-contingent aftereffect due to color adaptation during inspection of the stimulus patterns. Perception and Psychophys ics, 11, 406-408.

Holding, O.H. (in press). McCollough effects as conditional responses: A reply to Skowbo. Perception and Psychophysics.

Hubel, D.H. \& Wiesel, T.N. (1968). Receptive fields and functional architecture of monkey striate cortex. Journal of Physiology, 195, 215-243.

Leppmann, P.K. (1973). Spatial frequency dependent chromatic aftereffects. Nature, 242, 411-412.

Long, T. (1984). Human factor principles for the design of computer color graphics displays. British Telecommunication Technological Journal, 2, 5-14.

Lovegrove, W.J. \& Over, R. (1972). Color adaptation of spatial frequency detectors in the human visual system. Science, 176, 541-543.

Lund, N.J., \& Mackay, D.M. (1983). Sleep and the McCollough effect. Vision Research, 23, 903-906.

Mackay, D.M. \& Mackay, Y. (1974). The trial course of the McCollough effect and its physiological implications. Journal of Physiology, 327, 38 .

McCollough, C. (1965). Color adaptation of edge detectors in the human visual system. Science, 149, 115-116.

Mikaelian, M. (1980). Effective ?uminance contract as a parameter in contingent aftereffects. Perception \& Psychophysics, 27, 531-536. 
Munse 11, A.H. (1941). Color Notation. Baltimore: Munsell Color Company.

Murch, G.M. (1975). Visual and Auditory Perception. New York: Bobbs-Merrill.

Murch, G.M. (1976). Classical conditioning of the McCollough effect: Temporal parameters. Vision Research, 16, 615-619.

Murch, G.M. (1.979). The role of test pattern background hue in the McCollough effect. Vision Research, 19, 939-94?.

Murch, G.M., \& Hirsch, J. (1972). The McCollough effect created by compl imentary afterimages. American Journal of Psychology, 85, 241247.

Riggs, L.A. (1973). Curvature as a feature of pattern vision. Science, 181, 1070-1072.

Riggs, L.A., White, K.D., \& Eimas, P.D. (1974). Establishment and decay of orientation-contingent aftereffects of color. Perception and Psychophysics, 16, 535-542.

Schmidt, M.J., Pinette, P. \& Finke, R. (1978). Further evidence for conditioning processes in the McCollough effect. Journal of General Psychology, 99, 117-132.

Schmidt, M.J., \& Finke, R. (1979). Contrast and frequency competition for orientation-contingent aftereffects of coior. Perception and Psychophysics, 25, 406-412.

Sharpe \& Tees. (1978). Contour specificity of the McCollough effect. Perception and Psychophysics, 23, 451-458.

Skowbo, D. (1984). Are the McCollough effects conditioned responses? Psychology Bulletin, 96, 215-226.

Skowbo, D., \& Forster. (1983). Further evidence against a classical conditioning model of McCollough effects. Perception and Psychophysics, 34, 552-554.

Skowbo, D., Gentry, T., Timney, B., \& Morant, R.B. (1974). The McCollough effect: influence of several $k$ inds of visual stimulation on decay rate. Perception and Psychophysics, 16, 47-49.

Skowbo, D., \&Rich, J. (1982). Practice does not facilitate acquisition of McCoillough effects: evidence against a learning model. Perception \& Psychophysics, 32, 551-554.

Skowbo, D., Timney, B., Gentry, T., \& Morant, R.B. (1975). McCollough effects: experimental findings. Psychology Bullet in, 82, 497-510.

Skowbo, D. \& White, K.D. (1983). McCollough effect acquisition depends on duration of exposure to inducing stimuli, not number of stimulus presentations. Perception and Psychophys ics, 34, 549-551. 
Stromeyer, C.F. (1969). Further studies of the McCoilough effect. Perception and Psychophysics, 6 , 105-110.

Stromeyer, C.F. (1972). Edge contingent color aftereffects, spatial frequency specificity. Vision research, 12, 717-732.

Stromeyer, C.F. (1974). Form-specific color aftereffects in scotopic illumination. Nature, 250, 266-268.

Stromeyer, C.F., \& Mansfield, R. (1970). Colored aftereffects produced with moving edges. Perception and Psychophysics, ?, 108-114.

Teft, L.W., \& Clark, F.T. (1968). The effects of stimulus density on orientation specific aftereffects of color adaptation. Psychonomic Science, 11, 265-266.

Timney, B., Gentry, T., Skowbo, D., \& Morant, R.B. (1974). Chromatic grating thresholds and the McCollough effect. Vision Research, 14, $1033-1035$.

Hasserman, G.S. (1978). Color vision: an historical introduction. New York: John Wiley and Sons.

White, K.D. (1976). Luminance as a parameter in establishmert and testing of the McCollough effect. Vision Research, 16, 297-302.

White, K.D., \& Riggs, L.A. (1974). Angle-contingent color aftereffects, Vision Research, 14, 1147-1.154.

Wolman, B.B. (1973). Dictionary of Behavioral Science. New York: Van Nostrand Reirhold Company. 